Vol. 2 Issue 1, Jan.2012, pp. 042-045

\title{
Reduction in Encoding time using Graph Based image Segmentation for fractal image compression
}

\author{
Payal D.Thakare \\ M-Tech Student Department of Computer \\ Technology \\ Y.C.C.E. Nagpur ,441110, India.
}

\author{
Shailesh D.Kamble \\ Department of Computer Technology \\ Y.C.C.E. Nagpur, 441110,India
}

\begin{abstract}
Fractal coding is a very promising technique for image compression. However, it has not been widely used because of its long encoding time and high computational complexity Fractal image compression has received much attention from the research community because of some desirable properties like resolution independence, fast decoding, and very competitive rate-distortion curves. Despite the advances made, the long computing times in the encoding phase still remain the main drawback of this technique. So far, many different solutions have been proposed for this problem, but there is not yet a standard for fractal coding. Based on adaptive threshold quad tree fractal compression approach, we consider image's semantic characteristic, and apply graph-based image segmentation to fractal image compression, separating the initial image into many logic areas, then encoding each area with fractal image compression method. According to the problem of long encoding time which exists in the typical fractal compression approaches, we have proposed a fast image fractal compression approach based on the combination of adaptive quad tree compression approach and graph-based image segmentation algorithm.
\end{abstract}

Keywords- Fractal compression, image compression, graph based image segmentation

\section{INTRODUCTION}

In Image Compression, the researchers' aim is to reduce the number of bits required to represent an image by removing the spatial and spectral redundancies. Recently discrete wavelet transform, wavelet packet, fractal coding has emerged as popular techniques for image compression. Image fractal compression is originated from Barnsley's research for IFS system and the image fractal block coding proposed by Jacquin . In 1988, Barnsley applied fractal image compression based on IFS system to computer graphics, and compressed the aerial image, which made him get a compressed ratio 1000:1, but the approach requires manual intervention. Subsequently, Jacquin proposed a new fractal image compression method based on image block, and the method can conduct automatically without manual intervention. Therefore, Jacquin's method has become a typical representation for this research direction; fractal image compression has become practical since then[1].

Although fractal compression has many advantages, but in the process of fractal encoding, it needs a lot of computing, and this results in a long encoding time. Currently, fractal compression's research focuses on how to shorten the encoding time[1].Reference [2] proposed an adaptive threshold quad tree fractal compression approach, Compared with fixed square segmentation compression approach, it has a greater flexibility. It divides the image block which has high details into smaller sub-block, and for the image block with low details, divides them into larger sub-block. By doing so, it can reduce the number of image blocks which need to match and shorten the encoding time. Reference [3] proposed a method which applies ant algorithm to fractal compression, and implements the automatic classification for image block. When matching, it can use heuristic information and substitute global search with local research. By the comparison of average brightness between image block and sub image block, reference [4] divides sub-block into 15 categories, further, by sorting the image block's variance, every category can be classified into 24 sub-classes. So, total image blocks can be divided into 360 categories, when matching, we search in the same category. Based on graph theory, reference [5] proposed a fast image segmentation algorithm, by comparing the characteristic difference between regions and interior domains, it can judge whether there is a boundary between the two regions. Compared with traditional image segmentation algorithm, this algorithm can obtain images' global visual features. In this paper, based on adaptive threshold quad tree fractal compression algorithm, we propose a method which can apply the image segmentation algorithm proposed in reference [5] to fractal compression.

Following are the disadvantages of the above approaches. Firstly, they only consider some statistic information of image block, and neglect the image block's semantics and the semantic relationship between block and block. For example, the image block from the image's background has little probability of matching the image block from image's main content; secondly, once a R block can't find the best-match D block in its own search space, it can't expand its search to his adjacent search space, therefore, the decoding image has poor quality. Section II will introduce the proposed approach. In Section III will introduce the experimental result. 


\section{Payal D.Thakare, Shailesh D.Kamble / IOSR Journal of Engineering (IOSRJEN) \\ $w w w . i o s r j e n . o r g$ \\ ISSN : 2250-3021}

Vol. 2 Issue 1, Jan.2012, pp. 042-045

\section{A. What is Fractal Image Compression?}

Imagine a special type of photocopying machine that reduces the image to be copied by half and reproduces it three times on the copy (see Figure 1). What happens when we feed the output of this machine back as input? Figure 2 shows several iterations of this process on several input images. We can observe that all the copies seem to converge to the same final image, the one in 2(c). Since the copying machine reduces the input image, any initial image placed on the copying machine will be reduced to a point as we repeatedly run the machine; in fact, it is only the position and the orientation of the copies that determines what the final image looks like.[6]

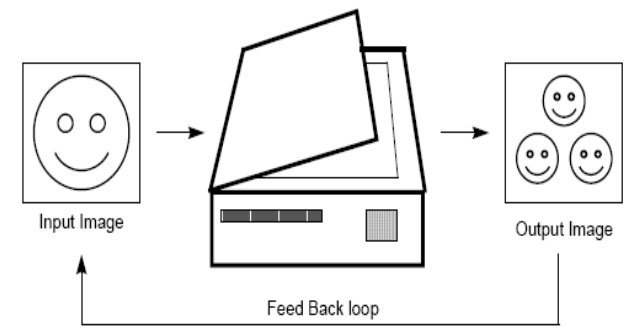

Figure 1. A copy machine that makes three reduced copies of the input image [8],[9].

The way the input image is transformed determines the final result when running the copy machine in a feedback loop. However we must constrain these transformations, with the limitation that the transformations must be contractive (see contractive box), that is, a given transformation applied to any two points in the input image must bring them closer in the copy. This technical condition is quite logical, since if points in the copy were spread out the final image would have to be of infinite size. Except for this condition the transformation can have any form.

In practice, choosing transformations of the form [6]

$$
w_{i}\left[\begin{array}{l}
x \\
y
\end{array}\right]=\left[\begin{array}{l}
a_{i} b_{i} \\
c_{i} d_{i}
\end{array}\right]\left[\begin{array}{l}
x \\
y
\end{array}\right]+\left[\begin{array}{l}
e_{i} \\
f_{i}
\end{array}\right]
$$

is sufficient to generate interesting transformations called affine transformations of the plane. Each can skew, stretch, rotate, scale and translate an input image. A common feature of these transformations that run in a loop back mode is that for a given initial image each image is formed from a transformed (and reduced) copies of itself, and hence it must have detail at every scale. That is, the images are fractals.


Figure 2. The first three copies generated on the copying machine Figure 1[7]

\section{B. Basic fractal image encoding approach}

The basic ideal of fractal image compression is as following: divide initial image into small image blocks with non-overlapping (Rang block, R block for short). For each $\mathrm{R}$ block, find an image block (Domain block, D block for short) which is the most similar to current $\mathrm{R}$ block under a certain transform, that is, use some image blocks' transformation to splice the initial image, and make the spliced image similar to the original image as much as possible[1].

Suppose the image which to be encoded is $256^{*} 256$ size with 256 gray shade and $\mathrm{R}$ block is a $8 * 8$ size block, so the whole image can be divided into $1024 \mathrm{R}$ blocks, all the $\mathrm{R}$ blocks composite $\mathrm{R}$ pool. Suppose $\mathrm{D}$ block is four times larger as $\mathrm{R}$ block, so the number of $\mathrm{D}$ block is $(256-2 * 8+1) 2=58081$, all the D blocks composite D pool. For each R block, find a D block from D pool which is the most similar to it. The concrete steps are as following:

1) Shrink D block to the size of R block, marked D' block, and the specific shrinking method is four neighborhoods regional method

2) Transpose, turn D' block. Specifically, we can choose eight affine transformations which proposed by Jacquin, and the corresponding transformation matrix.

3) Compare each R block with all D' blocks in D pool, and obtain the most similar D' block. The similarity can be measured with average variance MSE, if we see each $\mathrm{R}$ block and D block as vectors.

4) For each R block, record the corresponding compression affine transformation $\mathrm{W}$. All compression affine transformations constitute the whole image's fractal code. 


\section{Payal D.Thakare, Shailesh D.Kamble / IOSR Journal of Engineering (IOSRJEN) \\ $w w w . i o s r j e n . o r g$ \\ ISSN : 2250-3021}

Vol. 2 Issue 1, Jan.2012, pp. 042-045

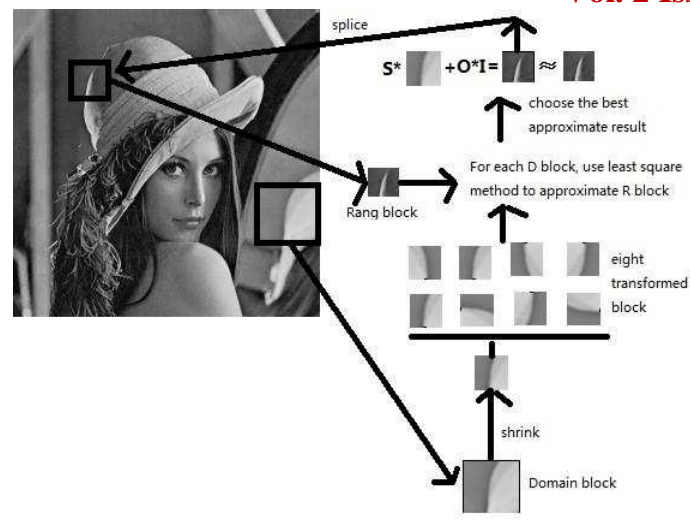

Figure 3.The schematic diagram of the fractal encoding process[1]

\section{PROPOSED APPROACH}

This paper proposes an image fractal compression approach based on the combination of adaptive quad tree compression approach and graph-based image segmentation, which will be used to reduce the encoding time and increase the compression ratio.

\section{A. Graph based image segmentation}

Graph-based image segmentation overcomes the shortcomings of traditional image segmentation algorithm, i.e. excessive segmentation over image. At the same time, this algorithm can adjust its segmentation scale according to different images, thus, it can achieve better content-based image segmentation. In the fractal encoding process, most blocks which come from different image's contents have different textures, so blocks come from different image contents can't be the best-match block for each other in most cases, but for the blocks come from the same image content, they can easily match each other well[1]. An example is shown in figure 4.

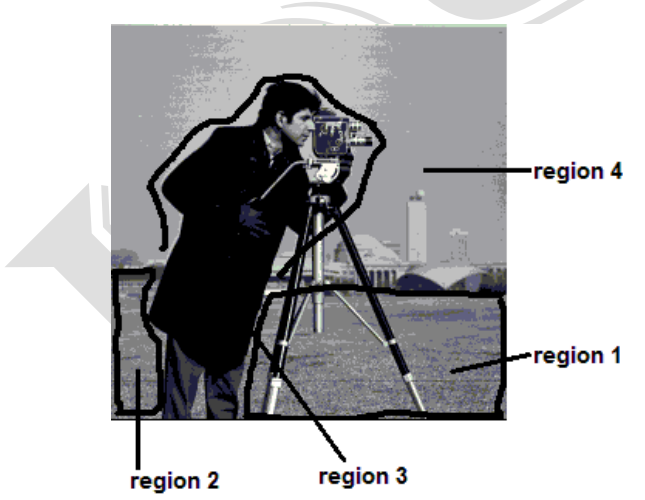

Figure 4. Schematic diagram of image content segmentation [1]

From figure 4, we can see that, blocks come from region 1 can easily find his best-match block in region 1 , the situation for blocks come from region 3 is the same as that in region 1, but blocks come from region 1 can hardly find his best-match block in region 3.Inspired by this fact, we combine graph- based image segmentation algorithm and adaptive threshold quad tree fractal compression approach together, and propose a new fractal image compression approach, use graph-based image segmentation approach to segment image at an appropriate segmentation scale, separate the original image into many different logic areas according to image content, and construct the corresponding search space for each logic area. Then encode each logic area using adaptive threshold quad tree approach. When the encoding for all logic areas in the whole image is finished, the whole image's encoding is completed. But after we separate the initial image into several different logic areas, it is difficult to encode the irregular shape with fractal compression approach; in this we adopt the following method [1].

After the initial image is separated into several different logic areas, we can set a same label for all pixels located in the same logic area, by doing so; we indicate that all those pixels are in the same logic area. When encoding, we can also set a label for each image block, and the label for image block is the equal to the labels of pixels located in the block, if there are several different pixels with different labels in one block, we can set the block's label as anyone of them, all the image blocks with the same label constitute a search space. As shown in figure 5, image block B can be either partitioned into the search space which A located in or partitioned into the search space which located in search space [1].

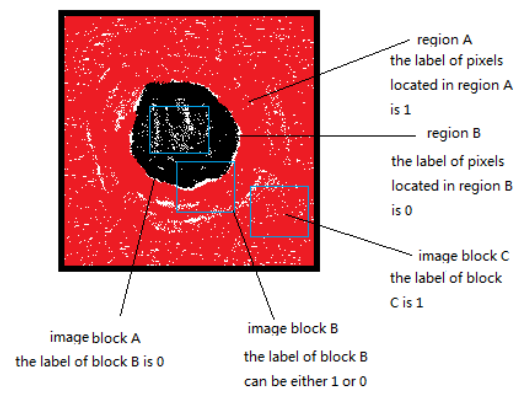

Figure 5. Schematic diagram of setting image block's label[1]

\section{EXPERIMENTAL SET UP AND RESULTS}

In our paper we take standard $256 * 256$ size, colored Lena image as test image. We have implemented to use the MATLAB 7.9 as a development tool on WINDOWS platform. Hardware requirement of proposed approach is general minimum configuration, Pentium III or above Processor, 256MB RAM (minimum), 400MB free disk space, etc.. The standard colored Lena image is shown in figure 6 , we have applied graph based image segmentation on original image then we got segmented image as shown in figure 7 , then labeling of each region has been done as shown in figure 8 . 
Payal D.Thakare, Shailesh D.Kamble / IOSR Journal of Engineering (IOSRJEN)

$w w w$.iosrjen.org

ISSN : 2250-3021

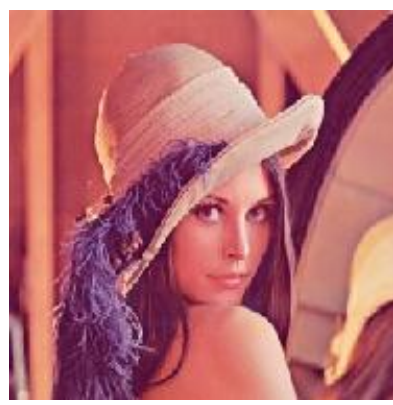

Vol. 2 Issue 1, Jan.2012, pp. 042-045

Figure 6 .Original Lina image

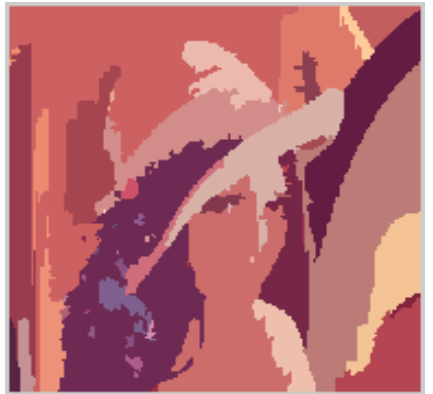

Figure 7.Lina image after segmentation

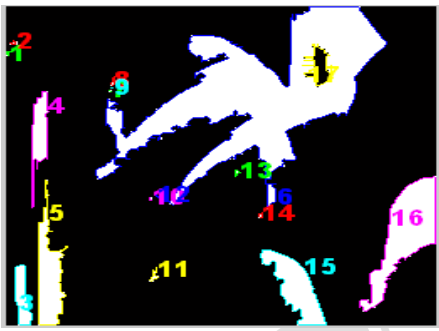

Figure8.Lina image after labeling to each region

\section{CONCLUSION}

We have considered image's semantic characteristic, and apply graph-based image segmentation to fractal image compression, separating the initial image into many logic areas. Proposed approach can improve the recovered image's quality and compression ratio significantly. This paper proposes an image fractal compression approach based on the combination of adaptive quad tree compression approach and graph-based image segmentation algorithm, which will be used to reduce the encoding time and increase the compression ratio.

In future work, we will implement an algorithm based on a graph based image segmentation and adoptive quad tree to reduce the encoding time and increase the compression ratio.

\section{REFERENCES}

[1] Hai Wang "Fast image fractal compression with graph based image segmentation algorithm" International Journal of Graphics Vol. 1, No.1, November, 2010

[2] Shiping Zhu,Liang Yu,Kamel Bellouata.”An improved fractal image coding algorithm based on adaptive threshold for quadtree partition". The International Society for Optical Engineering, v 7129, 2008
[3] Jinjiang Li, Da Yuan, Qingsong Xie, Caiming Zhang. "Fractal Image Compression by Ant Colony Algorithm". The 9th International Conference for Young Computer Scientists

[4] B.Hurtgen,C.Stiler,'Fast hierarchical codebook search for fractal coding of still images[J]",Proceedings of EOS/SPIE Visual Communications PACS Medical Applications'93,Berlin,1993:397 408.

[5] Pedro F. Felzenszwalb and Daniel P.Huttenlocher .'Efficient GraphBased Image Segmentation." International Journal of Computer Vision, 59(2) September 2004

[6] "An Introduction to fractal Image compression" Texas instrument Europe October 1997.

[7] Miroslav Galabov"Fractal Image Compression "International conference on computer syatem and technology 2003.

[8] Y.Fisher,'Fractal image compression".siggraph 92 course notes.

[9] Y.Fisher,'Fractal Image Compression:Theory of Application"springerverlag(1994)

[10] Davoine F,Antonini M,Chassery J.Barlaud M. "Fractal image compression based on delauney triangulation and vector quantization"'[J],IEEE Transactions $\quad$ Image Processing, 1996,5(2):338 346.

[11] LiWenjing,Li Wangchao."A fast fractal image coding technique[J].'Proceedings of ICSP'98:775 778.

[12] Macro Polvere,Michele Nappi."Speed-up in Fractal Image Coding:Comparison of Methods[J]".IEEE Transactions on Image Compression 2000,9(6):1002 1009.

[13] Raouf Hamzaoui, Dietmar Saupe, and Michael Hiller." Distortion Minimization with Fast Local Searchfor Fractal Image Compression." Journal of Visual Communication and Image Representation 12,450468 (2001)

[14] S. A. Curtis and C. E. Martin," Functional Fractal Image Compression"

[15] Miroslav Galabov."Fractal Image Compression", International Conference on Computer Systems and Technologies CompSysTech'2003

[16] Fisher,Y.,E.W.Jacobs,R.D.Boss.”Fractal Image Compression Using Iterated Transforms". Kluwer Academic Publishers, Norwall, 1989.

[17] Jacquin,"A.Fractal Theory of Iterated Markov Operators with Applications to Digital" Image Coding". Doctoral Thesis, Georgia Institute of Technology,1989.

[18] P.F. Felzenszwalb and D.P. Huttenlocher." Efficient graph-based image segmentation". Int. J. of Comp. Vis., 59(2):167-181, 2004.

[19] J. Shi and J. Malik." Normalized cuts and image segmentation". IEEE Trans. Pattern Anal. And Machine Intell., 22(8):888-905, 2000.

[20] Cochran, W.O,Hart, J.C,Flynn. P.J. Fractal volume compression. IEEE Transactions on Visualization and Computer Graphics, v 2, n 4, p 313322, Dec 1996

[21] Raouf Hamzaoui, Dietmar Saupe, and Michael Hiller. "Distortion Minimization with Fast Local Searchfor Fractal Image Compression”. Journal of Visual Communication and Image Representation 12, 450$468(2001)$

[22] Davoine F,Antonini M,Chassery J.Barlaud M.’Fractal image compression based on delauney Triangulation and vector quantization[J]",IEEE Transactions Image Processing,1996,5(2):338 346.

[23] Hurd,L.P,Gustavus,M.A,Barnsley,M.F.etc.”Fractal video compression. Compcon Spring '92'. Thirty-Seventh IEEE Computer Society International Conference, Digest of Papers. 